$19727 \mathrm{p}$ STARS K. Sakurai (NAS OF

KUNITOMO SAKURAI

\title{
GSFC GODDARD SPACE FLIGHT CENTER
}

GREENBELT, MARYLAND

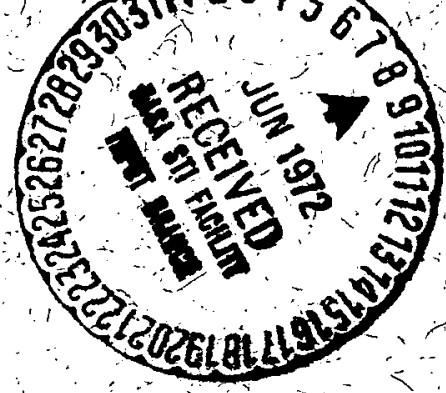


THE EFFECT OF THE CORIOLIS FORCE ON THE STABILITY OF ROTATING MAGNETIC STARS

by

\author{
Kunitomo Sakurai* \\ Radio Astronomy Branch \\ Laboratory for Extraterrestrial Physics \\ NASA/Goddard Space Flight Center \\ - Greenbelt, Maryland 20771
}

\begin{abstract}
The effect of the Coriolis force on the stability of rotating magnetic stars in hydrostatic equilibrium is investigated by using the method of the energy principle. It is shown that this effect is to inhibit the onset of instability.
\end{abstract}

*NASA Associate with University of Maryland 
Recently, the role of the Coriolis force has been studied on the dynamo processes related to the maintenance of magnetic fields of stellar objects like the sun and the earth (e.g., Parker, 1955; Steinbeck and Kraus, 1969).

These processes are necessarily associated with convective motions in the interior of stellar objects. In this paper, by using the method of the energy principle, we will consider the stability of rotating magnetic systems which are initially in hydrostatic equilibrium.

The energy principle which was first studied by Bernstein et al. (1958) will be extended to the rotating hydromagnetic systems. In dealing with the stability of such systems, the effect of the Coriolis force will necessarily be considered. In this case, in comparison with the case as considered by Bernstein et al. (1958), the momentum equation has an additional term which results from the existence of this force. Thus, this equation is given by

$$
\rho \frac{\partial^{2} \vec{\eta}}{\partial t^{2}}+2 \rho \vec{\Omega} \times \frac{\partial \vec{\eta}}{\partial t}=F(\vec{\eta})
$$

and 


$$
\begin{aligned}
F(\vec{\eta})= & \operatorname{grad}[\gamma P \operatorname{div} \vec{\eta}+(\vec{\eta} \cdot \operatorname{grad}) P]+\vec{j} \times \vec{Q} \\
& -\vec{B} \times \operatorname{curl} \vec{Q}+[\operatorname{div}(\rho \vec{\eta})] \operatorname{grad} \phi,
\end{aligned}
$$

where $\vec{\eta}, \vec{\Omega}, \mathbf{t}, \phi, \gamma, \mathbf{P}, \vec{j}$ and $\rho$ are the displacement vector due to perturbation, the angular velocity, the time, the gravitational potential, the ratio of specific heats, the pressure, the current intensity and the mass density, respectively. Here $\vec{Q}(\vec{\eta})$ is given by

$$
\vec{Q}(\vec{\eta})=\operatorname{cur1}(\vec{\eta} \times \vec{B})
$$

The second term on the left-hand side of (1) expresses the effect of the Coriolis force. This equation was first derived by Steinitz (1965). If we assume that $\vec{\Omega}=0$, this equation is reduced to (2-29) in the paper of Bernstein et al. (1958).

In order to study the stability of the systems as expressed by (1) and (2), we here assume that $\vec{\eta}$ is given by

$$
\vec{\eta}=\vec{\eta}_{0}(\vec{r}) e^{i \omega t}
$$

where $\vec{\eta}_{0}(\vec{r})$ is a complex displacement vector. By substituting (3) into (1), we obtain

$$
-\omega^{2} \rho \vec{\eta}+2 i w \rho(\vec{\Omega} \times \vec{\eta})=F(\vec{\eta})
$$


By multiplying scalarly $\vec{\eta}^{*}$ (a complex conjugate of $\vec{\eta}$ ) with

(4), we further obtain

$$
-\omega^{2} \rho \vec{\eta}^{*} \vec{\eta}+2 i \rho \vec{\eta}^{*}(\vec{\Omega} \times \vec{\eta})=\vec{\eta}^{*} \cdot \mathbf{F}(\vec{\eta})
$$

By taking into account the appropriate boundary conditions as given by Bernstein et al. (1958), this equation is integrated over the volume, in which the system under consideration is enclosed. Thus we obtain

$$
\begin{gathered}
-\omega^{2} \int \rho \vec{\eta}^{*} \vec{\eta} \mathbf{d v}+2 \omega \int \rho \vec{\eta}^{*} 1(\vec{\Omega} \times \vec{\eta}) \mathrm{dv} \\
=\int \vec{\eta}^{*} \cdot \mathrm{F}(\vec{\eta}) \mathrm{dv}
\end{gathered}
$$

Since both the right-hand side and the second term of the left-hand side of the above equation are real (Sakurai, 1972), we can solve this equation algebraically.

In order that the system is unstable, solution for $w$ must be complex. This condition is given as

$$
\left[\int \rho \vec{\eta}^{*} i(\vec{\Omega} \times \vec{\eta}) \mathrm{dv}\right] 2 / \int \rho \vec{\eta}^{*} \vec{\eta} \mathrm{dv}<\int \vec{\eta}^{*} \cdot \mathbf{F}(\vec{\eta}) \mathrm{dv}
$$

Since the left-hand side of (7) is positive, this condition represents that the system is stable in the domain $(-\infty, A)$, where

$$
\mathbf{A}=\left[\int \rho \vec{\eta}^{*} \mathbf{i}(\vec{\Omega} \mathbf{x} \cdot \vec{\eta}) \mathrm{dv}\right] \mathbf{2} / \int \rho \vec{\eta}^{*} \vec{\eta} \mathrm{dv}
$$


In the case that $\vec{\Omega}=0$, it follows that $A=0$. Therefore, this system is unstable whenever the following inequality is fulfilled:

$$
\vec{\eta}^{*} \cdot \mathbf{F}(\vec{\eta}) \mathrm{dv}>0
$$

Hence the domain in which the system is unstable is $(0, \infty)$. As has been deduced from (7), the domain, in which the system for $\vec{\Omega} \neq 0$ is unstable, is given by $(A, \infty)$, where $A>0$. This result indicates that the effect of the Coriolis force is to stabilize the system and then to inhibit the onset of fluid motion inside the system. Since the number A involves the factor $\Omega^{2}$, the effect of the coriolis force becomes stronger as the magnitude of $\vec{\Omega}$ increases.

In the case which the system fulfilles the condition (7), $\omega$ is complex and so the system is necessarily over-stable. This means that, in this system, fluid motion grows oscillatorilly as a result of over-stability.

As has been mentioned previously in this paper, the system, in general, is stabilized as a result of the action of the Coriolis force: the effect of this force is to inhibit the onset of convection, which is generated as a result of instability. It is said, therefore, that the 
effect of this force plays an important part in the study of fluid motion inside the rotating magnetic stars. In fact, since the dynamo action for the maintenance of their magnetic fields is deeply connected with the onset of fluid motion inside those stars, it seems important to examine the effect of the Coriolis force on the onset of over-stability as regards the convection inside the rotating magnetic stars. 